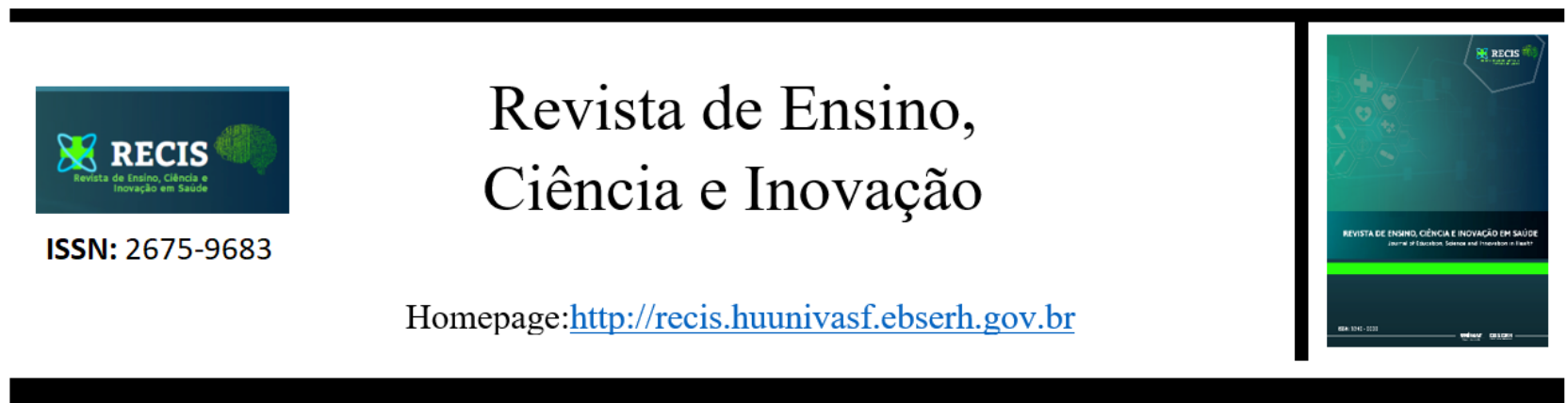

\title{
Hanseníase: estudo epidemiológico no município de Juazeiro-BA (2018-2020)
}

\section{Leprosy: epidemiological study in the city of Juazeiro-BA (2018-2020)}

\section{Carolline Xavier de Aguiar ${ }^{1}$, Jorge Welison Pereira Barbosa ${ }^{1}$, Renan Clementino de Oliveira ${ }^{1}$, Samuel Carvalho Silva ${ }^{1}$, Drako de Amorim Souza ${ }^{1}$, Tânia Rita Moreno de Oliveira Fernandes $^{2}$}

\author{
${ }^{1}$ Discente, Colegiado de Medicina, Campus Petrolina. ${ }^{2}$ Docente, Colegiado de Medicina, Campus Petrolina. \\ Universidade Federal do Vale do São Francisco (Univasf).
}

Autor correspondente: carollxavier3@gmail.com

Artigo recebido em 29/01/2021 e aceito em 17/03/2021

\begin{abstract}
RESUMO
Esse estudo teve como objetivo descrever indicadores epidemiológicos e aspectos clínicos dos novos casos de hanseníase no município de Juazeiro-BA. Trata-se de um estudo ecológico, abrangendo o período de 2018 a 2020. Foram notificados em Juazeiro, 370 novos casos. Nota-se uma redução do número de casos ao longo dos anos. Em relação às características epidemiológicas dos casos notificados, houve maior frequência entre sujeitos do sexo masculino, em níveis de escolaridade mais baixos, faixa etária mais acometida de 40 a 59 anos, forma clínica mais identificada foi a dimorfa e o grau de incapacidade física zero foi o predominante. Também, observa-se, uma taxa média de detecção independente da faixa etária de 56,53/100.000 habitantes, colocando o município na classificação de hiperendêmico. No que concerne à taxa de detecção em menores de 15 anos, é considerada alta, 2,9/100.000 habitantes, demonstrando redução em comparação aos estudos anteriores. Os dados apresentados neste estudo revelam que os casos novos de hanseníase ocorridos em Juazeiro-BA entre 2018 a 2020 possuem características similares às observadas nacionalmente. O empenho das políticas públicas e inclusivas devem visar a divulgação e elucidação dos casos, na tentativa de solucionar as iniquidades em saúde, tendo em vista a complexidade em curso, o da hiperendemia.
\end{abstract}

Palavras-chave: Hanseníase; Doenças negligenciadas; Epidemiologia.

\begin{abstract}
This study aimed to describe epidemiological indicators and clinical aspects of new leprosy cases in the city of JuazeiroBA. This is an ecological study, covering the period from 2018 to 2020. In Juazeiro, 370 new cases were reported. There has been a reduction in the number of cases over the years. Regarding the epidemiological characteristics of the reported cases, there was a higher frequency among male subjects, at lower levels of education, the most affected age group from 40 to 59 years, the most identified clinical form was the dimorphic and the degree of zero physical disability was the predominant one. Also, it is observed, an average detection rate independent of the age group of 56.53 / 100,000 inhabitants, placing the municipality in the classification of hyper endemic. With regard to the detection rate in children under 15, it is considered high, 2.9 / 100,000 inhabitants, showing a reduction compared to previous studies. The data presented in this study reveal that the new cases of leprosy that occurred in Juazeiro-BA between 2018 to 2020 have characteristics similar to those observed nationally. The commitment of public and inclusive policies must aim at the dissemination and clarification of cases, in an attempt to solve health inequities, in view of the ongoing complexity, that of hyper-endemic.
\end{abstract}

Keywords: Leprosy; Neglected Diseases; Epidemiology. 


\section{INTRODUÇÃO}

A hanseníase é uma doença infectocontagiosa, de caráter crônico, ocasionada pelo Mycobacterium leprae, que acomete os nervos periféricos e, especialmente, as células de Schwann. Transmissível pelas vias respiratórias através de contato próximo e prolongado de pessoas suscetíveis com um doente que não está recebendo terapia adequada. Trata-se de uma das enfermidades mais antigas que assolam a humanidade e, atualmente, um dos maiores problemas de saúde pública no país. Manifesta-se por meio de lesões dermatoneurológicas que cursam de forma lenta e progressiva, podendo levar à incapacidade física, social, psicológica e econômica. . $2,3,4,5,6,7,8,9,10$

Ainda que após a instituição da Poliquimioterapia (PQT), em 1991, como tratamento específico tenha resultado na eliminação da doença como um problema de saúde pública global em 2000, anualmente, em média, mais de 200 mil casos novos ainda são reportados à Organização Mundial da Saúde (OMS), afirmando-se como um desafio a ser superado. ${ }^{6,711,12,13,14,15,16}$ Nesse sentido, diferentes estratégias têm sido empreendidas pela OMS com objetivo de alcançar o controle da hanseníase, entre elas a Estratégia Global para a Hanseníase 20162020 que é voltada, principalmente, para os países que até então não conseguiram alcançar a meta proposta de menos de 1 caso de hanseníase por 10 mil habitantes. ${ }^{1,8,16,17,18}$

Em 2017, a OMS publicou registro epidemiológico que apontava o Sudeste Asiático e as Américas como as áreas mais afetadas pela doença. $^{14,19}$ Esse evento ocorre, sobretudo, em países em desenvolvimento, demonstrando relação íntima com as condições ambientais e socioeconômicas. ${ }^{7,20} \mathrm{Na}$ última década, 22 países foram listados como de prioridade global de acordo com a tendência de detecção de novos casos. Entre eles, estão Índia, Brasil e Indonésia, que juntos representaram $80,2 \%$ da carga de novos casos mundial. ${ }^{19}$

No território brasileiro, também em 2017, foram diagnosticados 26.875 novos casos de hanseníase, representando $92,35 \%$ dos casos reportados nas Américas. ${ }^{19}$ De modo epidemiológico, o país apresenta alta endemicidade, com coeficiente de detecção anual de 12,23 casos por 100 mil habitantes, ocupando o segundo lugar em números absolutos de casos novos da hanseníase, atrás apenas da Índia. ${ }^{1,19,21,22,23}$ Assim, ao longo das últimas décadas, o Ministério da Saúde vem empreendendo esforços por meio de estratégias de controle da hanseníase baseadas, sobretudo, em detecção precoce, início imediato, adesão e conclusão do tratamento, prevenção e manejo das incapacidades, vigilância dos contatos e educação em saúde..$^{24,25}$

Contudo, a habilidade na operacionalização dessas ações associadas aos determinantes imunológicos, genéticos e sociais de cada região, refletem-se na distribuição da disseminação da infecção de modo heterogêneo pelo Brasil. ${ }^{14,15,26}$ Por conseguinte, estudos evidenciam que as regiões do país que concentram os níveis mais elevados de endemicidade são as regiões Norte, Centro-Oeste e Nordeste. ${ }^{20,26}$ Conquanto a região Nordeste seja a terceira em coeficiente de detecção na população geral, constituiu $43,5 \%$ de todos os casos de infectados do país em 2016. ${ }^{15}$

O Estado da Bahia (BA), maior estado do Nordeste, acompanha essa mesma circunstância epidemiológica de endemicidade, apresentando em 2017 coeficiente de detecção de 14,5 casos por 100 mil habitantes. ${ }^{18,20,27}$ No período de 2007 a 2017, foram notificados no município baiano de Juazeiro 1.561 novos casos, com coeficiente médio de detecção equivalente a 65 casos por 100 mil habitantes. ${ }^{28}$ Nesse contexto, a cidade foi classificada como um dos 253 municípios prioritários no Brasil, conforme o Plano Integrado de Ações Estratégicas 2011-2015 do Ministério da Saúde, por meio da Portaria 2.556, de 28 de outubro de 2011.2,24,29 Entretanto, o coeficiente de detecção na população permaneceu muito alto mesmo após a implementação dessas estratégias, de modo que em 2017 a taxa de detecção de 51,9 por 100 mil habitantes, demonstrando não ter conseguido eliminar a hanseníase como um problema de saúde pública. ${ }^{28}$

Considerando que a hanseníase é uma enfermidade de grande importância para a saúde pública, a análise de fatores individuais e coletivos como idade, sexo, escolaridade, condições socioeconômicas, clínicas e geográficas, ao mesmo tempo em que tornam complexo o serviço de vigilância, são cruciais como fonte de planejamento das intervenções a serem estabelecidas em um território. Logo, a relevância desse estudo se dá pela escassez de pesquisas mais recentes sobre o município que apurem esse fenômeno de adoecimento com base nesses indicadores que tanto ampliam o conhecimento científico e contribuem na elaboração de ações de controle da doença. ${ }^{13,20,23,24,25,30,31}$

Posto isso, o presente estudo teve como objetivo descrever indicadores epidemiológicos e aspectos clínicos dos casos novos de hanseníase no 
município de Juazeiro, Bahia, no triênio de 2018 a 2020 .

\section{MATERIAL E MÉTODOS}

Trata-se de um estudo epidemiológico, observacional, analítico, ecológico e retrospectivo, abrangendo o período de 2018 a 2020. A coleta de dados foi realizada a partir do Sistema de Informação de Agravos de Notificação (SINAN). As variáveis analisadas foram: data da notificação, sexo, faixa etária, escolaridade, forma clínica e Grau de Incapacidade Física (GIF).

Juazeiro é um município brasileiro com área territorial de $6.721,237 \mathrm{~km}^{2} \mathrm{e}$ população estimada de 218.162 habitantes, ocupando a quinta posição entre as cidades mais habitadas do estado, localizado na Mesorregião do Vale do São Francisco, no norte da Bahia que, por sua vez, possui área territorial de $564.760,427 \mathrm{~km}^{2} \mathrm{e}$ 14.930.634 habitantes. ${ }^{32,33} \mathrm{O}$ tamanho da população residente estimada foi proveniente das projeções intercensitárias produzidas pelo Instituto Brasileiro de Geografia e Estatística (IBGE) para o ano de 2020.

Como critério de inclusão, foram considerados todos os casos novos confirmados e notificados de hanseníase na cidade de Juazeiro (BA), de 2018 a 2020. Foi realizada uma análise exploratória e descritiva de todos os dados relativos aos indicadores sociais e clínicos, através do auxílio do software Excel 2016.

$\mathrm{O}$ indicador epidemiológico utilizado foi o coeficiente de incidência de hanseníase/100.000 habitantes. ${ }^{24}$

$\mathrm{O}$ presente estudo foi realizado exclusivamente com dados secundários, sem identificação dos sujeitos, obedecendo aos princípios éticos conforme as resoluções 466/2012 e 510/2016 do Conselho Nacional de Saúde, o que justifica a ausência do parecer de Comitê de Ética em Pesquisa em Seres Humanos. ${ }^{34}$

\section{RESULTADOS}

Entre 2018 e 2020, foram notificados, em Juazeiro, 370 casos da doença. Em geral, nota-se uma redução do número de casos absolutos ao longo dos anos em estudo (165 casos em 2018, 135 em 2019 e 70 em 2020), exibindo uma média de, aproximadamente, 123,33 casos por ano.

Essa redução se mostrou expressiva quando comparada à variável sexo, conforme pode ser visualizada na Figura 1 apontando, em 2018, 90 casos em indivíduos do sexo masculino $(54,55 \%)$ e 75 casos em indivíduos do sexo feminino $(45,45 \%)$ e, em 2020, 49 em indivíduos do sexo masculino $(70,00 \%)$ e 21 indivíduos do sexo feminino $(30,00 \%)$.

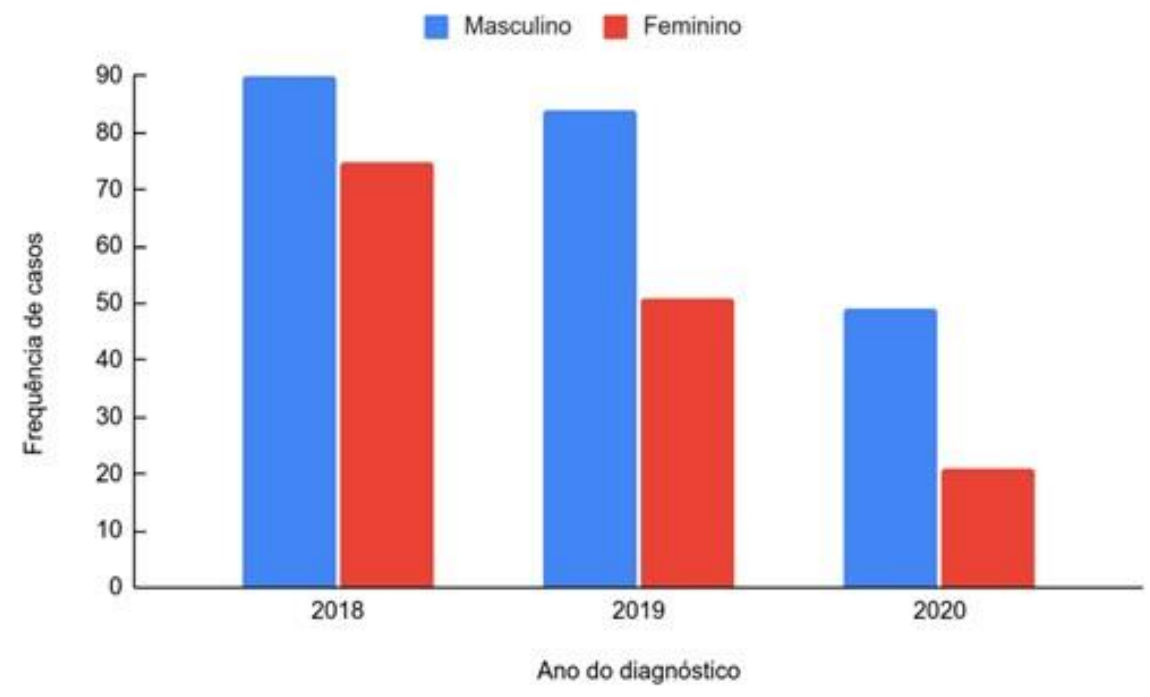

Figura 1. Notificações segundo o sexo, $M$ (masculino) e F (feminino), a cada ano.

Em relação às características coletadas dos novos casos, de acordo com a Tabela 1, houve maior concentração no sexo masculino (223/60,27\%). Quanto à escolaridade, notou-se maior incidência nos níveis de escolaridade mais baixos $(269 / 72,70 \%)$, com predominância no ensino fundamental 2 incompleto $(80 / 21,62 \%)$ e maior incidência entre jovens e adultos em idade economicamente ativa $(230 / 62,16 \%)$, trazendo a faixa etária de 40 a 59 anos como a mais acometida $(146 / 39,46 \%)$. 
Revista de Ensino, Ciência e Inovação em Saúde v.2, n.1 (2021) 18-26

Tabela 1. Indicadores epidemiológicos e aspectos clínicos da hanseníase em Juazeiro (BA), de 2018 a 2020

\begin{tabular}{|c|c|c|}
\hline Variáveis & $\mathbf{N}$ & $\%$ \\
\hline \multicolumn{3}{|l|}{ Sexo } \\
\hline Masculino & 223 & $60,27 \%$ \\
\hline Feminino & 147 & $39,73 \%$ \\
\hline \multicolumn{3}{|l|}{ Escolaridade } \\
\hline Ignorados* & 34 & $9,19 \%$ \\
\hline Analfabeto & 43 & $11,62 \%$ \\
\hline 1 - Ensino fundamental 1 incompleto & 72 & $19,46 \%$ \\
\hline 2 - Ensino fundamental 1 completo & 29 & $7,84 \%$ \\
\hline 3 - Ensino fundamental 2 incompleto & 80 & $21,62 \%$ \\
\hline 4 - Ensino fundamental completo & 19 & $5,14 \%$ \\
\hline 5 - Ensino médio incompleto & 26 & $7,03 \%$ \\
\hline 6 - Ensino médio completo & 46 & $12,43 \%$ \\
\hline 7 - Educação superior incompleta & 8 & $2,16 \%$ \\
\hline 8 - Educação superior completa & 10 & $2,70 \%$ \\
\hline Não se aplica & 3 & $0,81 \%$ \\
\hline \multicolumn{3}{|l|}{ Idade } \\
\hline 1 a 14 anos & 19 & $5,14 \%$ \\
\hline 15 a 39 anos & 97 & $26,22 \%$ \\
\hline 40 a 59 anos & 146 & $39,46 \%$ \\
\hline 60 anos ou mais & 108 & $29,19 \%$ \\
\hline \multicolumn{3}{|l|}{ Forma Clínica } \\
\hline Ignorados* & 6 & $1,62 \%$ \\
\hline Indeterminada & 21 & $5,68 \%$ \\
\hline Tuberculóide & 94 & $25,41 \%$ \\
\hline Dimorfa & 176 & $47,57 \%$ \\
\hline Virchowiana & 73 & $19,73 \%$ \\
\hline \multicolumn{3}{|l|}{ Grau de Incapacidade Física } \\
\hline Em branco & 1 & $0,27 \%$ \\
\hline Grau Zero & 265 & $71,62 \%$ \\
\hline Grau I & 45 & $12,16 \%$ \\
\hline Grau II & 38 & $10,27 \%$ \\
\hline Não Avaliado & 21 & $5,68 \%$ \\
\hline
\end{tabular}

* Dados que não foram preenchidos na Ficha de Notificação/Investigação, ou que foram preenchidos como "Ignorado" 
No que tange às características clínicas em análise, conforme a Figura 2, sobressai-se a forma Dimorfa $(176 / 47,57 \%)$ seguida da Tuberculóide $(94 / 25,41 \%), \quad$ Virchowiana $\quad(73 / 19,73 \%) \quad$ e
Indeterminada $(21 / 5,68 \%)$, respectivamente. Em 6 casos $(1,62 \%)$ esse dado não foi considerado na ficha de notificação sendo, portanto, não classificado.

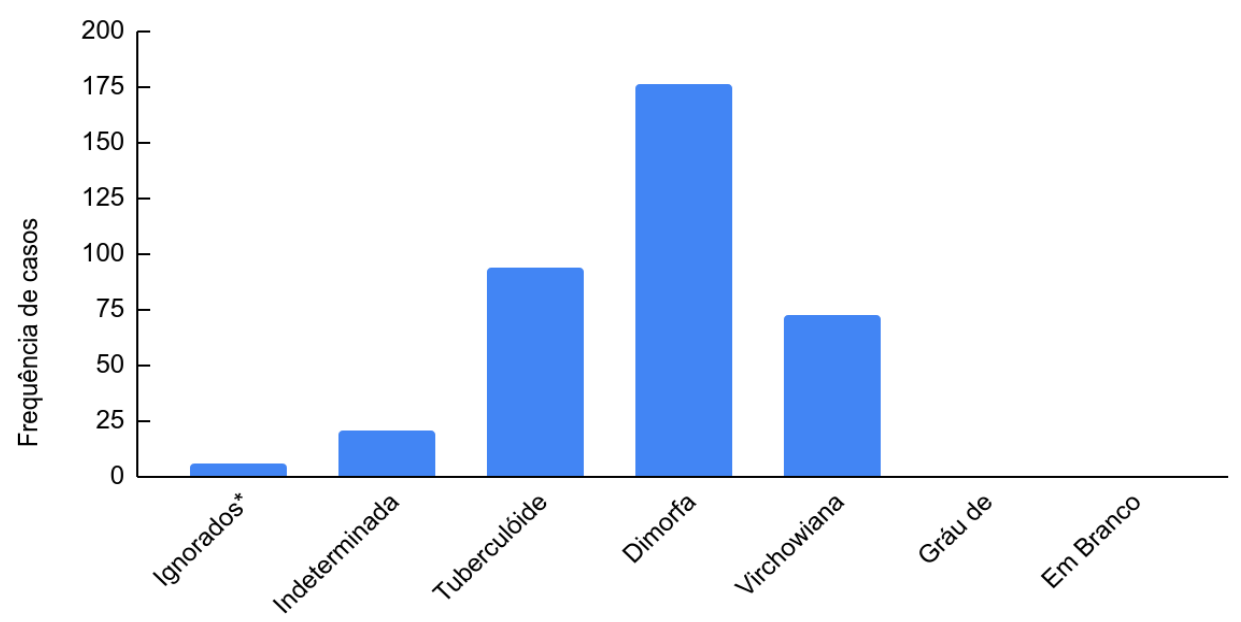

Forma Clínica

Figura 2. Notificações por forma clínica.

Ao correlacionar formas clínicas com grau de incapacidade física, mediante a Figura 3, observou-se predominância de Grau Zero em todas as formas, chegando a um total de 265 casos (71,62\%), como mostra (Tabela 1). Em seguida, o Grau 1 de incapacidade física demonstrou-se como o segundo maior em número de notificações nas formas Tuberculóide $(8 / 2,16 \%)$ e Dimorfa $(28 / 7,57 \%)$. Por sua vez, a forma clínica Virchowiana apresentou Grau 2 de incapacidade física como segundo maior número de notificações (17/4,59\%). Em 21 casos (5,68\%) o GIF não foi avaliado.

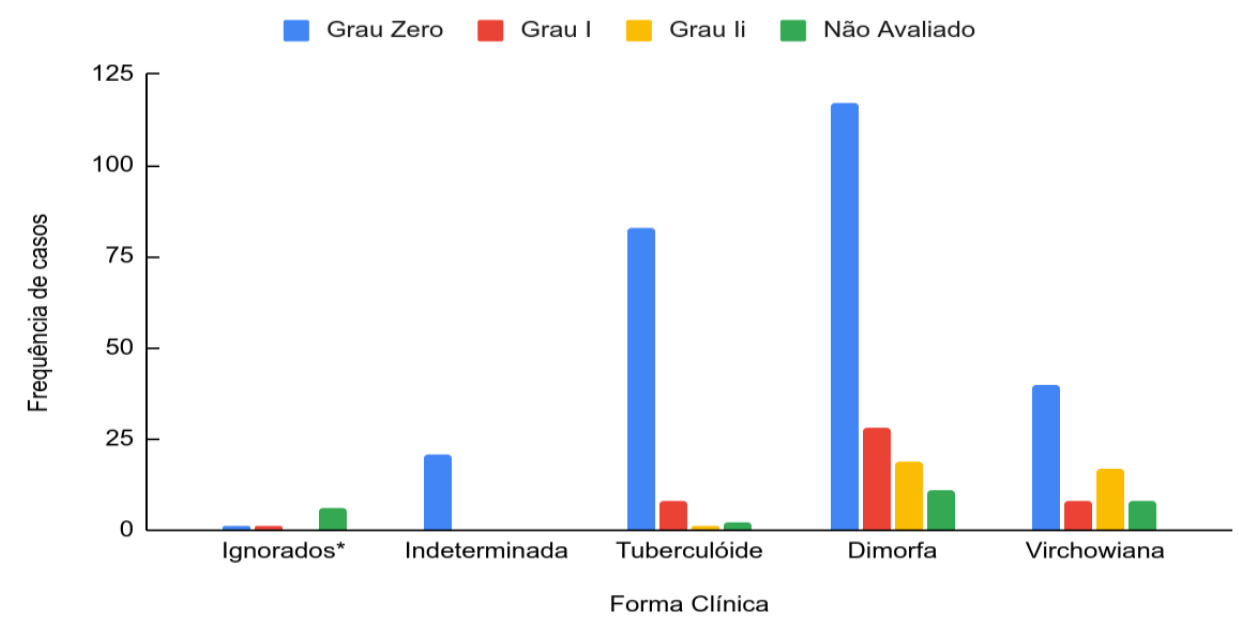

Figura 3. Relação entre formas clínicas e GIF

\section{DISCUSSÃO}

Os dados apresentados neste estudo revelam que os casos novos de hanseníase ocorridos na cidade de Juazeiro (BA) entre os anos de 2018 a 2020 possuem características similares às observadas nacionalmente. No ano de 2020, 
constatou-se uma redução expressiva no número de notificações, 70 novos casos, quando em 2019 foram 135 e em 2018 foram notificados 165 casos novos. Vale ressaltar que o baixo número de notificações no ano de 2020 reflete o atual momento de pandemia pela COVID-19 que atravessa o país, que resultou em menor procura aos serviços de saúde em casos de doenças com menor gravidade aparente. ${ }^{35}$

No Brasil, há uma maior notificação de casos de hanseníase entre o sexo masculino nos últimos anos $(55,2 \%) .{ }^{23}$ Em Juazeiro ocorre algo similar, porém com uma taxa mais elevada $(60,27 \%)$. Por entender que esses sujeitos são maioria dos casos novos, assim como, procuram menos os serviços de saúde se comparados aos indivíduos do sexo feminino, além das questões culturais e impossibilidades de acesso e atenção à saúde que também são responsáveis por esse resultado, que o Ministério da Saúde publicou nota informativa despertando sobre a importância da investigação clínica qualificada, busca ativa e sensibilização de agendamentos em horários alternativos, como medidas de controle, quebra de cadeia de transmissão e prevenção de incapacidades físicas, muito relacionados a esse gênero. ${ }^{36}$

Em relação à escolaridade, pacientes analfabetos correspondem a 11,62\% das notificações do período. Ademais, observa-se grande parte dos casos em pessoas com ensino fundamental incompleto, somatório dos dados 1,2 e 3 da variável escolaridade na Tabela 1, apresentando 48,91\% dos casos totais. Ao comparar com dados do boletim nacional de hanseníase de 2020, notamos outra similaridade, haja vista que $43,3 \%$ dos pacientes possuem ensino fundamental incompleto. ${ }^{23} \mathrm{~A}$ incidência elevada entre pacientes analfabetos ou com ensino fundamental incompleto pode ser justificada por um menor grau de conhecimento sobre formas de prevenção e sobre a importância de acompanhamento de saúde rotineiro, ${ }^{37}$ enquanto a prevalência e possível agravamento do quadro estão ligados a uma baixa adesão e continuidade ao tratamento, que pode ser justificada pela descrença no diagnóstico, efeitos colaterais das medicações ou a crença em uma cura espontânea. ${ }^{38}$

Também em relação à variável escolaridade, $10 \%$ dos dados não puderam ser avaliados adequadamente. Em 2020, o Ministério da Saúde, tal qual o presente estudo, apontou uma taxa considerável de dados não fornecidos no país $(17,6 \%) .{ }^{23}$ Quanto a isso, é essencial ressaltar a importância do preenchimento adequado da ficha de notificação, para evitar subnotificações ou preenchimento com alguns dados incorretos/ausentes. Com o preenchimento adequado da ficha, é possível ter uma noção mais realista da saúde no Brasil, possibilitando melhor direcionamento das verbas provenientes do governo. Portanto, é fundamental a educação permanente dos profissionais de saúde, que são os responsáveis pela alimentação do SINAN. ${ }^{12,13,23}$

No que concerne à idade, a faixa etária mais prevalente foi a de 40 a 59 anos, simbolizando $39,46 \%$ dos casos. Em paralelo, 36,97\% dos pacientes tinham a faixa etária supracitada, em relação aos casos notificados entre 2014-2018 no Brasil. ${ }^{23}$ Em pacientes com menos de 15 anos, foram notificados 19 casos nos três anos de estudo, equivalente a $5,14 \%$ dos casos totais, percentual proporcional comparado a um estudo anterior na mesma cidade, que relatou $7,8 \%$ dos casos entre menores de 15 anos. Os impactos da faixa etária nos estudos epidemiológicos salientam sua importância na cadeia de transmissão da doença. ${ }^{2}$

A taxa de detecção por 100.000 habitantes, independentemente da faixa etária, foi de 56,53, o que coloca Juazeiro (BA) como um município hiperendêmico por ter mais de 40 casos por 100.000 habitantes, conforme parâmetros do Ministério da Saúde., ${ }^{2,24}$ Por sua vez, entre notificados com menos de 15 anos, o coeficiente de detecção foi de 2,9/100.000, podendo ser classificado, de acordo com os parâmetros do Ministério da Saúde, como uma taxa de detecção "alta" (de 2,50 a 4,99 por 100.000 habitantes), confirmando uma redução expressiva em oposição aos dados de estudo anterior, que constatou taxa superior a 10/100.000 habitantes, considerada como "hiperendêmico" $(\geq 10$ por 100.000) também conforme o parâmetro anteriormente citado.,23

A forma clínica mais diagnosticada entre 2018 e 2020 foi a Dimorfa, representando 47,57\% dos casos notificados nesse período, o que corrobora com informações relatadas em outro estudo sobre hanseníase, no qual a forma dimorfa correspondeu a 42,4\%. ${ }^{11}$ Aspectos relacionados à faixa etária, moradia e educação, representam fatores de risco entre os contatos das formas multibacilares, sendo fundamental a sorologia para o rastreio entre os envolvidos. ${ }^{39}$

Por conseguinte, quanto ao GIF, houve dominância de Grau Zero (265/71,62\%), seguidos de Grau $1(45 / 12,16 \%)$ e Grau 2 (38/10,27\%), respectivamente. Quanto à relação entre o GIF e a forma clínica dos sujeitos, as formas multibacilares apresentaram relação direta com graus maiores de incapacidade física. Esse evento exibe o nível da qualidade de cobertura dos serviços de saúde (classificado como "regular" conforme o Ministério da Saúde), a precocidade do diagnóstico (tendo em vista os menores graus de incapacidade 
se apresentarem mais prevalentes) e auxilia no planejamento de ações a nível de atenção primária em saúde. ${ }^{23,40}$

\section{CONCLUSÃO}

Este estudo atualiza os dados dos casos de hanseníase na cidade de Juazeiro (BA), sob o enfoque de diversos fatores sociais e características clínicas inerentes a esta patologia.

Os resultados mostram uma redução no número de casos ao longo do triênio avaliado, com um decréscimo ainda maior no ano de 2020, o que mantém relação direta com o cenário atual no qual o mundo tem atravessado, tendo o foco maior para a pandemia do coronavírus em detrimento de outras comorbidades.

Em relação às variáveis, nota-se que o número de casos tem presença maior no público masculino, em pacientes com menor nível de instrução, na população jovem e adulta economicamente ativa, na faixa etária adulta focada entre 40 e 59 anos e a forma clínica mais presente nos novos casos, a Dimorfa; sendo observado uma similaridade com o padrão nacional quando comparado com análise da literatura.

Com isso, é importante frisar a relevância das políticas públicas em saúde com enfoque na promoção da saúde e na prevenção de agravos para melhor elucidação dos determinantes sociais em saúde e seu impacto na população; bem como a educação em saúde, tanto a permanente quanto a continuada, para a adequada divulgação dos dados não apenas da hanseníase, mas das diversas doenças de notificação compulsória e investigação obrigatória, em atenção, as negligenciadas, visto que o município em estudo é considerado hiperendêmico para a doença.

Propõe-se com este estudo trazer reflexões e um olhar mais cuidadoso e direcionado para os casos de agravos notificáveis, em foco a hanseníase, para que estratégias e políticas sociais e inclusivas sejam realizadas para a minimização das iniquidades em saúde, trabalhando dessa forma a equidade e por conseguinte, o controle desta hiperendemia.

\section{REFERÊNCIAS}

1. Jardim CP, Santos HLPC, Araújo VMA, et al. Análise da produção científica brasileira sobre hanseníase identificada na base de dados web of science. Arq. Cienc. Saúde UNIPAR. 2020;24:105-111.

https://doi.org/10.25110/arqsaude.v24i2.2020.770 9

2. Souza CDF, Lima RS. Endemia hansênica em menores de 15 anos e a ampliação da Atenção
Primária no município em Juazeiro, Bahia: estudo de séries temporais. Rev. APS. 2019;22(2):300318. 8363.2019.v22.15897

https://doi.org/10.34019/1809-

3. Souza CDF, Fernandes TRMO, Matos TS, et al. Grau de incapacidade física na população idosa afetada pela hanseníase no estado da Bahia, Brasil. Acta Fisiatr. 2017;24(1):27-32. https://doi.org/10.5123/S1679-

49742020000400006

4. Loures LF, Mármora $\mathrm{CHC}$, Barreto J, et al. Percepção do estigma e repercussões sociais em indivíduos com hanseníase. Psicologia em Estudo. 2016;21(4):665-675.

https://doi.org/10.4025/psicolestud.v21i4.30037

5. Souza EA, Heukelbach J, Oliveira MLWDR, et al. Baixo desempenho de indicadores operacionais de controle da hanseníase no estado da Bahia: padrões espaçotemporais, 2001-2014. Rev Bras Epidemiol.

2020;23:e200019. https://doi.org/10.1590/1980-549720200019

6. Araújo KMFA, Lana FCF. Relação da hanseníase com a cobertura da estratégia saúde da família e condições socioeconômicas. Ciencia y enfermeria.

2020;26(1).

http://dx.doi.org/10.4067/s0717-

95532020000100201

7. Leano HAM, Araújo KMFA, Bueno IC, et al. Fatores socioeconômicos relacionados à hanseníase: revisão integrativa da literatura. Rev Bras Enferm. 2019;72(5):1474-1485. http://dx.doi.org/10.1590/0034-7167-2017-0651

8. Santos KCB, Corrêa RGCF, Rolim ILTP, et al. Estratégias de controle e vigilância de contatos de hanseníase: revisão integrativa. Saúde debate. 2019;43(121):576-591.

http://dx.doi.org/10.1590/0103-1104201912122

9. Ribeiro MDA, Silva JCA, Oliveira SB. Estudo epidemiológico da hanseníase no Brasil: reflexão sobre as metas de eliminação. Rev Panam Salud Publica. 2018;42:e42. https://doi.org/10.26633/RPSP.2018.42

10. Ministério da Saúde (BR). Secretaria de Vigilância em Saúde. Departamento de Vigilância das Doenças Transmissíveis. Guia prático sobre a hanseníase / Ministério da Saúde, Secretaria de Vigilância em Saúde, Departamento de Vigilância das Doenças Transmissíveis. Brasília: Ministério da Saúde; 2017. 68p.:il. Disponível em: https://portalarquivos2.saude.gov.br/images/pdf/2 017/novembro/22/Guia-Pratico-de-HanseniaseWEB.pdf

11. Sousa CRS, Feitosal MCR, Pinheiro ABF, et al. Aspectos epidemiológicos da hanseníase em um município nordestino do Brasil. Rev Bras Promoç Saúde. 2019;32:9469. https://doi.org/10.5020/18061230.2019.9469 
12. Leano HAM. Análise epidemiológica da hanseníase no nordeste brasileiro: vulnerabilidade individual, programática e social. Tese (Doutorado em Enfermagem). Belo Horizonte (MG): Escola de Enfermagem da Universidade Federal de Minas Gerais; 2019.130p. Disponível em: https://repositorio.ufmg.br/bitstream/1843/30800/ 1/TESE_HELOISYMEDEIROSLEANO.pdf

13. Souza CDF, Medronho RA, Santos FGB, et al. Modelagem espacial da hanseníase no estado da Bahia, Brasil, (2001-2015) e determinantes sociais da saúde. Ciênc. saúde coletiva. 2020;25(8):2915$2926 . \quad$ http://dx.doi.org/10.1590/141381232020258.21522018

14. Rodrigues RN, Leano HAM, Bueno IC, et al. High-risk areas of leprosy in Brazil between 20012015. Rev Bras Enferm. 2020;73(3):e20180583. http://dx.doi.org/10.1590/0034-7167-2018-0583

15. Souza CDF, Luna CF, Magalhães MAFM. Transmissão da hanseníase na Bahia, 2001-2015: modelagem a partir de regressão por pontos de inflexão e estatística de varredura espacial. Epidemiol. Serv. Saúde. 2019;28(1):e2018065. https://doi.org/10.5123/s1679-

49742019000100015

16. World Health Organization (WHO). Global Leprosy Strategy: Accelerating towards a leprosyfree world. World: World Health Organization. 2016. 36 p. Disponível em: https://apps.who.int/iris/bitstream/handle/10665/2 08824/9789290225201-pt.pdf

17. Souza EA, Ferreira AF, Pinto MSAP, et al. Desempenho da vigilância de contatos de casos de hanseníase: uma análise espaço-temporal no Estado da Bahia, Região Nordeste do Brasil. Cad. Saúde Pública. 2019;35(9):e00209518. http://dx.doi.org/10.1590/0102-311x00209518

18. Souza EA, Boigny RN, Ferreira AF, et al. Vulnerabilidade programática no controle da hanseníase: padrões na perspectiva de gênero no Estado da Bahia, Brasil. Cad. Saúde Pública. 2018;34(1):e00196216.

http://dx.doi.org/10.1590/0102-311x00196216

19. World Health Organization (WHO). Organisation mondiale de la Santé. (2018). Global leprosy update, 2017: reducing the disease burden due to leprosy. Situation de la lèpre dans le monde, 2017: reduction de la charge de morbidité due à la lèpre. Weekly Epidemiological Record = Relevé épidémiologique hebdomadaire, 93(35): 445-456. World Health Organization = Organisation mondiale de la Santé. Disponível em: https://apps.who.int/iris/bitstream/handle/10665/2 74290/WER9335-445-

456.pdf? sequence $=1 \&$ isAllowed $=\mathrm{y}$

20. Azevedo YP, Bisco VAS, Oliveira RI, et al. Perfil epidemiológico e distribuição espacial da hanseníase em Paulo Afonso, Bahia. Rev baiana enferm. 2021;35: e37805. http://dx.doi.org/10.18471/rbe.v35.37805

21. Souza RG, Lanza FM, Souza RS. Sensibilização dos Agentes Comunitários de Saúde para a atuação nas ações prevenção e controle da hanseníase: relato de experiência. HU Revista. 2018;44(3):411-415.

https://doi.org/10.34019/1982-

8047.2018.v44.25621

22. Vieira NF, Martínez-Riera JR, Lana FCF. Primary care quality and its effects on leprosy monitoring indicators. Rev Bras Enferm. 2020;73(4):e20190038.

http://dx.doi.org/10.1590/0034-7167-2019-0038

23. Ministério da Saúde (BR). Secretaria de Vigilância em Saúde. Departamento de Doenças de Condições Crônicas e Infecções Sexualmente Transmissíveis. Boletim epidemiológico da hanseníase 2020. Brasília: Ministério da Saúde; Número Especial |jan. 2020. 52 p. Disponível em: http://www.aids.gov.br/pt-br/pub/2020/boletimepidemiologico-de-hanseniase-2020

24. Ministério da Saúde (BR). Secretaria de Vigilância em Saúde. Departamento de Vigilância das Doenças Transmissíveis. Plano integrado de ações estratégicas de eliminação da hanseníase, filariose, esquistossomose e oncocercose como problema de saúde pública, tracoma como causa de cegueira e controle das geohelmintíases: plano de ação 2011-2015. 1. ed., 1. reimpr. Brasília: Ministério da Saúde, 2013. 100 p.: il. Disponível em:

http://bvs.saude.gov.br/bvs/publicacoes/plano_int egrado_acoes_estrategicas_hanseniase.pd

25. Ministério da Saúde (BR). Secretaria de Vigilância em Saúde. Departamento de Doenças de Condições Crônicas e Infecções Sexualmente Transmissíveis. Estratégia Nacional para Enfrentamento da Hanseníase 20192022/Ministério da Saúde, Secretaria de Vigilância em Saúde, Departamento de Doenças de Condições Crônicas e Infecções Sexualmente Transmissíveis. Brasília: Ministério da Saúde, 2019. 109 p.: il. Disponível em: http://portalarquivos2.saude.gov.br/images/pdf/20 19/marco/27/Estrategia-Nacional-CGHDE-

Consulta-Publica-27mar.pdf

26. Boigny RN, Souza EA, Ferreira AF, et al. Falhas operacionais no controle da hanseníase em redes de convívio domiciliar com sobreposição de casos em áreas endêmicas no Brasil. Epidemiol. Serv. Saúde. 2020;29(4): e2019465. https://doi.org/10.5123/s1679-

49742020000400004

27. Secretaria de Saúde do estado da Bahia. Diretoria de Vigilância Epidemiológica-Divep 
Revista de Ensino, Ciência e Inovação em Saúde v.2, n.1 (2021) 18-26

Boletim Epidemiológico de Hanseníase/2018. 2018; (1): 1-5. Disponível em: https://www.saude.ba.gov.br/wpcontent/uploads/2017/11/boletimEpidemiogicoHa nseniaseAgo2018_n\%C2\%BA01.pdf

28. Fernandes TRMO, Pereira AA, Cardoso LS, et al. The hidden prevalence of leprosy: a comparative study between two Brazilian cities. Rev. Assoc. Med. Bras. 2020;66(10): 1338-1343. http://dx.doi.org/10.1590/1806-9282.66.10.1338 29. Ministério da Saúde (BR). Portaria n ${ }^{\circ} 2.556$, de 28 de outubro de 2011. Estabelece mecanismo de repasse financeiro do Fundo Nacional de Saúde aos Fundos de Saúde Estaduais, do Distrito Federal e Municipais, por meio do Piso Variável de Vigilância e Promoção da Saúde, para implantação, implementação e fortalecimento da Vigilância Epidemiológica de Hanseníase, Tracoma, Esquistossomose e Geohelmintíases. Diário Oficial da União. Brasília (DF), 2011 out $11 . \quad$ Disponível em: http://www.saude.campinas.sp.gov.br/saude/lista legislacoes/legis_2012/PT-MS-GM-

2556_281011.pdf

30. Silva JSHA, Mota JC, Silva RS, et al. Descrição dos registros repetidos no Sistema de Informação de Agravos de Notificação, Brasil, 2008-2009. Epidemiol. Serv. Saúde. 2016;25(3):487-498.

http://dx.doi.org/10.5123/S1679-

49742016000300005

31. Souza HP, Oliveira WTGH, Santos JPC, et al. Doenças infecciosas e parasitárias no Brasil de 2010 a 2017: aspectos para vigilância em saúde. Rev Panam Salud Publica. 2020;44:e10. https://doi.org/10.26633/RPSP.2020.10

32. Instituto Brasileiro de Geografia e Estatística (IBGE). Cidades e estados: juazeiro. 2020. Disponível em: https://www.ibge.gov.br/cidadese-estados/ba/juazeiro.html

33. Instituto Brasileiro de Geografia e Estatística (IBGE). Cidades e estados: bahia. 2020. Disponível em: https://www.ibge.gov.br/cidadese-estados/ba.html
34. Giovanella L, Martufi V, Mendoza DCR, et al. A contribuição da atenção primária à saúde na rede SUS de enfrentamento à Covid-19. Scielo preprints, $2020 \quad$ out 01. https://doi.org/10.1590/SciELOPreprints. 1286 35. Ministério da Saúde (BR). Conselho Nacional de Saúde. Resolução $n^{\circ}$ 466, de 12 de dezembro de 2012. Trata de pesquisas em seres humanos e atualiza a resolução 196. Diário Oficial da União. Brasília (DF), 2012 dez 12. Disponível em: https://bvsms.saude.gov.br/bvs/saudelegis/cns/201 3/res0466_12_12_2012.html

36. Ministério da Saúde (BR). Secretaria de Vigilância em Saúde. Departamento de Vigilância das Doenças Transmissíveis. Nota informativa conjunta ${ }^{\circ} 01$, de 2016 CGHDE/DEVIT/SVS/MS, COSAPI/DAPES/SAS/MS

CGGAB/DAB/SAS/MS. Brasília: Ministério da Saúde, 2016 ag. (1). Disponível em: https://central3.to.gov.br/arquivo/344609/

37. Silva MEGC, Souza CDF, Silva SPC, et al. Epidemiological aspects of leprosy in JuazeiroBA, from 2002 to 2012. An Bras Dermatol. 2015;90(6):799-805.

http://dx.doi.org/10.1590/abd1806-

4841.201533963

38. Luna IT, Beserra EP, Alves MDS, et al. Adesão ao tratamento da Hanseníase: dificuldades inerentes aos portadores. Brasília: Rev. bras. enferm.

2010;63(6).

https://doi.org/10.1590/S0034-

71672010000600018

39. Cunha MHCM, Silvestre MPSA, Silva AR, et al. Fatores de risco em contatos intradomiciliares de pacientes com hanseníase utilizando variáveis clínicas, sociodemográficas e laboratoriais. Rev Pan-Amaz Saud. 2017 jun;8(2):21-8. http://dx.doi.org/10.5123/S2176-

62232017000200003

40. Uchôa REMN, Brito KKG, Santana EMF et al. Perfil clínico e incapacidades físicas em pacientes com hanseníase. REUOL. 2017;11(Supl. 3):146472. http://doi.org/10.5205/reuol.10263-91568-1RV.1103sup201719 